\title{
DEAD-TIME COMPENSATORS: A UNIFIED APPROACH
}

\author{
Julio E. Normey-Rico* Eduardo F. Camacho** \\ * Dpto. Automação e Sistemas. Univ. Fed. de Sta Catarina, Brasil \\ ** Depto. Ing. Sist. y Automática, Univ. de Sevilla, España. \\ E-mails: julio@cartuja.us.es, eduardo@cartuja.us.es
}

\begin{abstract}
This paper shows how most dead-time compensators can be considered as a particular case of a proposed general control structure. The proposed structure can be tuned taking into account the performance and robustness of the closed-loop. The obtained controller is more general and allows better results than previous algorithms. In order to illustrate the results, some simulation examples are shown. Copyright $\odot 1998$ IFAC
\end{abstract}

Keywords: predictors, robustness, dead time systems.

\section{INTRODUCTION}

The dynamic behaviour of many industrial processes can be represented by models consisting of a differential equation and a dead-time. When the process exhibits long dead-time the performance of classical controllers deteriorates. In these cases, the use of a dead-time compensator in the structure of the controller can be the most efficient solution.

The first dead-time compensator (DTC), the Smith Predictor (SP), was proposed by Smith (1958) at the end of the 50's. Since then numerous extensions and modifications of the SP have been proposed. Watanabe and Ito (1981) proposed a DTC which could yield zero steady state error and desired transient responses to step disturbances showing that the SP cannot cope with step disturbances when the plant has an integral mode. In later papers several algorithms were proposed to improve the regulatory capabilities of the SP (Palmor and Powers (1985), Palmor (1986)); or to improve the set-point and disturbance response when controlling integrative plants with long dead time (Astrom et al. (1994), Matausek and Micic (1996)). Also in a recent paper (Hagglung (1996)) a three parameter SP is proposed that provides an easy way to tune the controller for industrial applications.

1 Work supported in part by CICYT Contract TAP and CAPES-BRASIL Contract BEXO448/95-6
Furukawa and Shimemura (1983) analysed the closed loop stability of the SP showing that the SP cannot be used with unstable plants. This result was also shown by Morari and Zafiriou (1989) using the IMC approach. The control schemes proposed in Furukawa and Shimemura (1983) and Watanabe and Ito (1981) allow an unstable plant to be controlled if a proper implementation of the predictor is used.

The robust stability of the SP has been studied in several works. In Palmor and Halevi (1983) the concept of practical unstability is used to show that if the primary controller is not properly tuned the SP could be unstable when small missmatch in the delay are considered, in spite of having good values of gain margin and phase margin for the ideal system. In Morari and Zafiriou (1989) the authors discuss some of the "myths" of SP, pointing out that the SP do not in any way increase sensitivity to modelling error; that is, the primary controller defines the robustness of the closed-loop. In the same work an IMC tuning of the SP is proposed taking into account robust performance. Also in Santacesaria and Scattolini (1993) and Palmor (1986) different tunings of the parameters of the SP are proposed in order to improve robustness. In a recent work Normey-Rico et al. (1997) a simple structure for improving the robustness of dead-time compensators is proposed which needs only three tuning parameters. 
In this paper some of the ideas presented in previous works are used to design a unified DTC taking into account the disturbance rejection characteristics, the robustness and the performance of the closed-loop.

The paper is organized as follows. Section 2 presents the unified structure for the DTC and the SP and some of its modifications are analysed as particular cases. Section 3 analyses the closedloop performance of the unified DTC and section 4 presents a design procedure. Some comparative simulations are shown in section 5 and finally the conclusions of the work are presented in section 6 .

\section{A GENERAL STRUCTURE FOR THE ANALYSIS OF THE DTC'S}

In general, the structure of a DTC includes a model of the process. This model is used to compute the prediction of the output of the plant. In the discrete case this prediction is computed using the actual and previous values of the output and input of the plant. So it is possible to represent the DTCs drawing a general block diagram, as shown in figure 1.

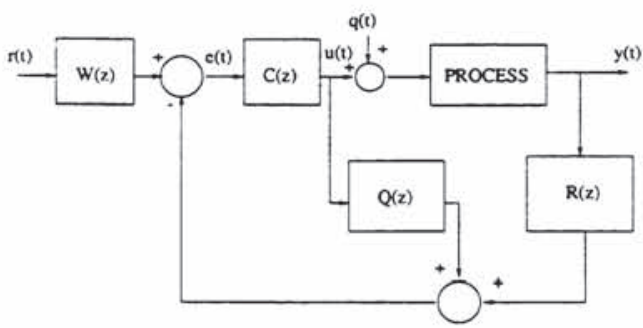

Fig. 1. Unified representation of the DTC's

In figure 1 blocks $R$ and $Q$ represent the predictor structure and blocks $C$ and $W$ represent the primary controller (a two-degree of freedom controller is normally used when good performance is desired, both for set-point and disturbance changes). This structure will be called unified dead-time compensator - UDTC. It is easy to show that the SP and its modifications can be derived from this general structure: (i) the SP is represented by the UDTC if $R=1$ and $Q=G_{n}$ $P_{n}$ where $P_{n}$ and $G_{n}$ represent, respectively, the model and delay free model of the plant; (ii) with the same relations but defining $Q=G_{1}-P_{n}$ this structure represents the DTC proposed by Watanabe and Ito (1981) where $G_{1}$ is computed in order to eliminate the poles of the plant from the minor loop; (iii) when $Q=G_{n}-P_{n}$ and $R$ is an approximated inverse of the dead-time, the UDTC is equivalent to the one proposed by Huang et al. (1990); (iv) with $Q=G_{n}-P_{n}$ and with $R$ a low pass filter the UDTC is the robust DTC presented in Normey-Rico et al. (1997), where $R$ is used to improve the robustness of the closed-loop.

Also other DTCs can be represented by the UDTC structure. Because of their practical interest the
DTCs proposed by Astrom et al. (1994) and Matausek and Micic (1996) to control integrative plants with long dead time are compared here. Although these two controllers use different tuning rules, they could be represented by the block diagram in figure 2.

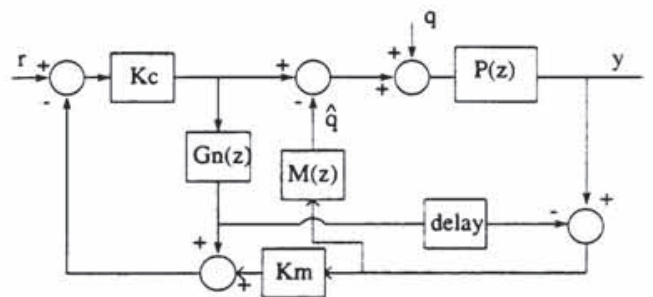

Fig. 2. DTCs by Astrom et al. $\left(K_{m}=0\right)$ and by Matausek and Micic $\left(K_{m}=1\right)$

Using block diagram transformations these structures can be drawn as in figure 1 with the following relation between blocks: (i) for the controller by Astrom et al. $Q=0, W=1, R=M$ and $C=K_{c}(1+$ $\left.P_{n} M\right) /\left(M+M K G_{n}\right)$ and (ii) for the controller by Matausek and Micic $W=1, C=K_{c}\left(1+M P_{n}\right)$, $Q=\left(G_{n}-P_{n}\right) /\left(1+P_{n} M\right)$ and $R=\left(1+M G_{n}+\right.$ $\left.M / K_{c}\right) /\left(1+P_{n} M\right)$.

Because of its characteristics, the proposed UDTC structure can be used to compute a controller taking into account the ideas given in NormeyRico et al. (1997) to increase the robustness, in Watanabe and Ito (1981) to cope with unstable plants and in Huang et al. (1990) to improve the disturbance rejection properties; but without the limitations of each of these previous algorithms, that cannot be applied to all types of plants or do not considered a complete set of specifications (robustness, disturbance rejection and performance).

\section{THE UDTC: CLOSED-LOOP ANALYSIS}

To analyse the closed-loop behaviour of the UDTC three transfer functions of the block diagram of figure 1 will be used: from the reference to the output $(L(z))$, from the disturbance to the output $(H(z))$ and from the disturbance to the control action $(U(z))$ :

$$
\begin{aligned}
& L(z)=\frac{W(z) C(z) P(z)}{1+C(z)(P(z) R(z)+Q(z))} \\
& H(z)=\frac{P(z)(1+C(z) Q(z))}{1+C(z)(P(z) R(z)+Q(z))} \\
& U(z)=\frac{-P(z) C(z) R(z)}{1+C(z)(P(z) R(z)+Q(z))}
\end{aligned}
$$

To analyse the robustness of the control system the plant will be represented by a transfer function $P(z)$ and unstructured uncertainties will be considered: $P=P_{n}(1+\delta P)$. If $d$ is the delay of the plant it is possible to write, in a discrete representation: $P=G z^{-d}$ and for the nominal case $P_{n}=G_{n} z^{-d_{n}}$. Thus $G$ represents the plant without the delay and $G_{n}$ is its nominal value. The norm-bound uncertainty region for $\delta P$ is 
computed in order to maintain closed-loop stability $(|\delta P(j \omega)| \leq \Delta P(j \omega)$, Morari and Zafiriou (1989)):

$$
\Delta P(j \omega)=\frac{\left|1+C\left(P_{n} R+Q\right)\right|}{|C R P|}(j \omega)
$$

with $\omega \in[0, \pi / T]$ ( $T$ is the sampling time). As the expression $\left(P_{n} R+Q\right)$ appears in the closed loop equations (1) and in the expression of the norm bound uncertainty region (2), the following parametrization is proposed:

Define two rational functions in $z, R$ and $X$ such that $Q=X-P_{n} R$, so $L_{n}, H_{n}, U_{n}$ and $\triangle P$ are given by:

$$
\begin{gathered}
L_{n}=\frac{C W P_{n}}{1+C X} \quad H_{n}=\frac{P_{n}(1+C Q)}{1+C X} \\
U_{n}=\frac{C R P_{n}}{1+C X} \quad \Delta P=\frac{|1+C X|}{\left|C R P_{n}\right|}=\frac{1}{\left|U_{n}\right|}
\end{gathered}
$$

In equations $3 L_{n}(z)$ defines the performance while $H_{n}(z)$ and $U_{n}(z)$ can be used to evaluate the disturbance rejection characteristics. Note that in $U_{n}(z)$ we can better appreciate the effect of the dead-time on the control action, and it is clear that an ideal $R(z)=z^{d}$ will cancel the effect of the dead-time (this is the idea used in Huang et al. (1990) to improve the disturbance rejection of the $\mathrm{SP})$. On the other hand, using $H(z)$ we can easily evaluate the effect of the poles of the plant in the disturbance response.

Also from equations (3) the following conclusions can be drawn:

- the nominal closed-loop transfer function, $L_{n}$, can be defined with an appropiate choice of $X, C$ and $W$

- with a defined $X, C$ and $W$, the uncertainty norm-bound, $\triangle P$, can be arbitrarily chosen with an appropiate $R$ and $L_{n}$ is not affected, so the robustness specifications and the nominal performance can be attempted independently (if the internal stability of the system is preserved).

- for good disturbance rejection performance it is desirable to have $U_{n}$ close to 1 at frequencies below the bandwidth of the closed-loop. As $\triangle P=\frac{1}{U_{n} \mid}, U_{n}$ cannot be defined independently from the robustness specifications.

- when the closed loop dynamic must be faster than the open loop one, $X$ must verify the relation $X=P_{n} Y\left(Q=P_{n}(Y-R)\right)$ in order to eliminate the open loop poles from the denominator of all the closed-loop transfer functions in equation (3). As the poles of $P$ are also poles of $Q$ the zeros of $Q$ must cancel these poles. On the other hand the poles of $R$ are always poles of $Q$, so if $R$ is chosen to improve the robustness (for instance as a low-pass filter) the disturbance rejection will be deteriorated. And if $R$ is used to improve the disturbance rejection of the controller then the system will be more sensitive to uncertainties.

- if $P$ is unstable then $X$ must cancel the unstable poles of $P$ in $H_{n}$ and $Q$ must be a stable transfer function. This condition guarantees the internal stability of the closed-loop as will be shown in the following.

\subsection{Internal Stability}

The general structure of the predictor based control system can be drawn in an alternative way, defining a cascade controller $K$ and a reference filter $S$ :

$$
K=\frac{C R}{1+C Q} \quad S=\frac{W}{R}
$$

thus, the internal stability of the control system can be analysed using the block diagram of figure 3 , where $q$ and $v$ are considered as exogenous disturbances. The denominator and numerator of the plant will be considered as $A$ and $B z^{-d}$ respectively; and $N_{i}$ and $D_{i}$ will represent the numerator and denominator of the others transfer functions.

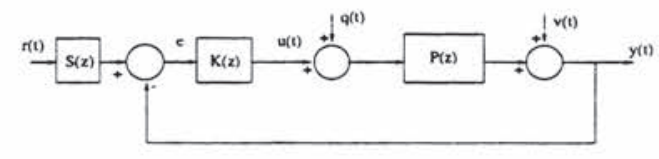

Fig. 3. Classical Representation of the UDTC

The necessary and sufficient condition for the internal stability of the system is that all the transfer functions between the inputs $r, v$ and $q$ and the outputs $e, u$ and $y$ are stable. Thus the polynomials $D_{q}, D_{r}, D_{w}$ and $D_{c} A+N_{c} B$ must have their roots inside the unitary circle. Considering that in every case it is possible to compute $C$ in order to stabilize the nominal plant without the delay (the roots of $D_{c} A+N_{c} B$ are positioned inside the unitary circle) and that $D_{r}$ and $D_{w}$ allways have their roots inside the unitary circle, $D_{q}$ will define the internal stability. Now it must be noted that for the SP and the DTCs proposed by Huang et al. (1990) and Normey-Rico et al. (1997) $D_{q}$ always have the same roots outside the unitary circle as $A$, so the closed loop will be internally stable if, and only if, the plant is stable or at the most has one pole in $z=1$. On the other hand, the DTC analysed in Watanabe and Ito (1981) has no roots outside the unitary circle. Now, in the UDTC the roots of $D_{q}$ must be inside the unitary circle. If the relation $Q=X-P_{n} R$ is used, then $X$ and $R$ must be chosen in order to cancel the unstable roots of $A$ in $D_{q}$. This solution is similar to the one proposed by Watanabe and Ito (1981) but in this case the inclusion of blocks $R$ and $W$ allows a design using stability, set-point and disturbance rejection performance and robustness criterions. 


\subsection{Disturbance rejection and robustness}

The modified versions of the SP can attempt better disturbance rejection than the original SP when the closed loop poles are considerably faster than the open loop ones or when the dead-time is small relative to the time constants of the plant, but this is only possible when plant uncertainties are small Palmor (1996). The same conclusions can be obtained using the structure of the UDTC noting that the transients of the responses of the system to step disturbances will depend on the defined closed loop poles (roots of $1+C X$ ) and on the roots of $D_{q}$. Suppose that $P$ has slow dynamics, thus if the poles of $P$ are roots of $D_{q}$ (as in the SP) these poles are poles of $H$ and so it is not possible to speed the transient of the disturbance response. This effect can be avoided if the slow poles of $P$ are not roots of $D_{q}$ in the UDTC.

Another way to improve the disturbance rejection of the DTC consists of the "elimination" of the dead-time between the disturbance and the control action (transfer function $U$ ) as proposed by Huang et al. (1990). Also in the UDTC the block $R$ can be computed in order to approximate the inverse of the dead time. Thus, the UDTC allows a general solution of the disturbance rejection problem because the design can take into account the advantages of the solutions proposed in previous works.

For stable plants with long dead-times, $R$ could be chosen as a low pass filter in order to improve the robustness (Normey-Rico et al. (1997)) and the tuning of this filter is straightforward, allowing its use in industrial applications.

In other cases, like in the observer predictor $\mathrm{Pal}$ mor (1996), the controller is computed to have a high disturbance rejection performance but obtaining a closed loop system considerably more sensitive to uncertainties because of the high pass characteristics of block $R$.

For the structure proposed in this paper $R$ corresponds to a low pass filter. If the plant is stable the cut-frequency of the filter must be chosen taking into account the compromise between disturbance rejection and robustness while $X, C$ and $W$ are used as performance parameters.

If the plant is unstable $X$ and $R$ must be chosen in order to have the denominator of $Q$ without roots outside the unitary circle. In this case the design of $C$ is more involved and it is not always possible to guarantee the existence of a solution with the desired performance and robustness. This case will be analysed in the next section where a general design procedure is presented.

From the previous analysis it follows that the proposed UDTC is a two degree of freedom control structure that can be used with stable or unstable plants and it allows the inclusion of robustness specifications in the design. So, it is more general than the SP and its modifications but has all the advantages of these type of controllers.

\section{UDTC: A DESIGN PROCEDURE}

In this section the best properties of each structure will be used in order to define a control scheme that presents the desired control specifications. The design procedure will first be presented for the general case. After that, and because of their practical importance, the stable plant case will be discussed.

\subsection{The general case}

The general predictor structure must be computed using the block diagram of figure 1 . In general the closed-loop specifications will include: internal stability, zero steady state error for constant inputs or disturbances, a desired set-point and disturbance response and robust stability.

To attemp the specifications $R, X, C$ and $W$ are chosen in order to satisfy the following relations:

- for the internal stability conditions $X$ must be chosen in order to have a stable $Q$

- to verify the steady state specifications: $R(1)=1$ and $\frac{C}{1+C Q}$ must have integral action. For the last condition it is sufficient to choose $C$ with integral action and $Q$ with a zero in $z=1$. In the general case $C$ will have $l$ integrators and $Q$ must have $l$ zeros in $z=1$.

- for the performance specifications a set of desired closed-loop poles must be defined

- for the robustness conditions $R$ must be a low pass filter, in general $R(z)=\left(\frac{1-\beta}{1-\beta z^{-1}}\right)^{\nu}$, $0<\beta<1$ and $\nu \geq 1$.

The denominator of the plant $A$ is factorized as $A=A_{u} A_{s}$ where $A_{s}$ has the poles inside the unitary circle, and $A_{u}$ is the unstable part of $A$.

Taking into account the previous conditions the following design procedure can be defined:

Step 1: Choose a value of $\beta$ and use $\nu=1$. Compute $R=N_{r} / D_{r}$.

Step 2: Define the denominator of $X$ as $D_{x}=A_{u}$

Step 3: Compute the numerator of $X\left(N_{x}\right)$ using the equation $X=Q+P_{n} R$ in order to obtain a stable $Q$ and to satisfy the steady state specifications:

$$
\frac{N_{q}}{D_{q}}=\frac{N_{x}}{D_{x}}-\frac{B N_{r} z^{-d}}{A D_{r}}=\frac{D_{r} A_{s} N_{x}-B N_{r} z^{-d}}{D_{r} A_{u} A_{s}}
$$

then $N_{x}$ must be chosen in order to make the polynomial $D_{r} A_{s} N_{x}-B N_{r} z^{-d}$ divisible by $A_{u}$ and $(z-1)^{l}$ where $l$ is the number of integrators of $C$. Note that with these conditions $N_{x}$ will have $l+\operatorname{gr}\left(A_{u}\right)$ coefficients, where $\operatorname{gr}\left(A_{u}\right)$ is the number of poles of $A_{u}$. Also note that the number 
of coefficients in $N_{x}$ does not depend on the order of $A_{s}$.

Step 4: Using $N_{x}$ and $D_{x}$ compute $C=N_{c} / D_{c}$ to solve the Diofantine equation $N_{x} N_{c}+D_{x} D_{c}=D_{d}$ where $D_{d}$ defines the desired poles.

Step 5: Compute filter $W$ in order to improve the set-point response.

Step 6: Test the robustness of the system using an estimation of the plant uncertainties. If the robust conditions are not verified then increase the value of $\beta$ and/or $\nu$ and go to step 2. When possible, iterate until the desired specifications are obtained.

Note that the solution of the problem is not guaranteed for every set of specifications. For unstable plants $R$ cannot be chosen as low pass as desired (in order to increase robustness) because some minimal feedback is necessary to maintain the closed-loop stability.

Another approach can be also used for the design of the UDTC instead of the solution of the previous polynomial diofantine equations. Using a frequency representation, $C, W, X$ and $R$ can be computed in order to obtain a desired shape for the closed loop transfer functions $L, H$ and $U$, and for the uncertainty norm boundary $\triangle P$.

\subsection{The stable plant case}

Many industrial processes are stable plants with dominant dead-times. For these plants a general rule is to retain the open-loop time constant of the process in the closed-loop system. Note that there is no sense in speeding up the closed-loop response in respect to the open-loop when the dead-time is dominant (Matausek and Micic (1996); Hagglung (1996)), and there are no advantages in the use of the modified structures of the SP such as the ones presented in Watanabe and Ito (1981)or Huang et al. (1990). In this case it is possible to choose $Q=P_{n}\left(z^{d}-R\right)$ maintaining internal stability and choosing $R$ as a low pass filter (in this case the nominal performance is defined by $X=G_{n}$ for every $R$ ). In this case the design procedure starts in step 4 where the new Diofantine equation is $N_{c} B+D_{c} A=D_{d}$

As $R$ must be computed in order to increase the norm bound uncertainty region at the desired frequency range, the choice of $C$ and $W$ must be made in parallel with $R$ in order to also attempt some dynamical and steady state specifications for the disturbance to output time response.

\section{SIMULATION EXAMPLES}

In this section two typical examples of the proposed method are presented.

Example 1: Stable case The model of the plant is given by $P(s)=\frac{k}{1+\tau s} e^{-t_{d}}$, where $k \in(0.8,1.2)$, $\tau \in(0.5,1.5)$ and $t_{d} \in(4,6)$. The nominal values of the parameters are: $t_{d}=5, k=1$ and $\tau=1$.

The controller must be computed in order to obtain (for the nominal case) a set point step response without overshoot and to maintain an aceptable response for all plants in the family. As the plant is stable it is possible to use $X=G_{n}$. A PI controller is computed using root locus: $C(z)=$ $\frac{0.4(z-0.7)}{z-1}, W(z)=\frac{0.3}{z-0.7}$ with sampling time $T=$ 0.2 . In figure 4 , the norm-bound uncertainty for $R=1$ (dotted line) and for $R(z)=\left(\frac{0.15}{z-0.85}\right)$ (dashed line) are compared to the unmodelled dynamics for different cases (solid line). Note that the system is robutly stable with $R=1$ but to maintain the closed-loop characteristics of the response a filter is used.

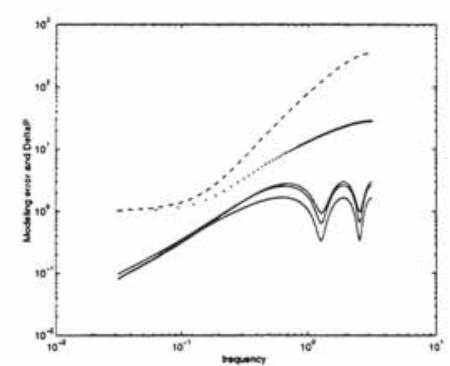

Fig. 4. Robustness analysis for example 1.

The closed loop behaviour of the closed loop system when parameter uncertainties are considered is shown in figure 5. At $t=0$ a step change in the reference is performed and at $t=50$ a $10 \%$ step disturbance is added at the input of the plant: case 1 is the nominal case (dashed-dotted line), case 2 is for $k=1.2, \tau=.6$ and $t_{d}=6$ (dashed line) case 3 is for $k=1.2, \tau=1.5$ and $t_{d}=4$ (solid line) and case 4 is for $k=.8, \tau=.5$ and $t_{d}=6$ (dotted line).

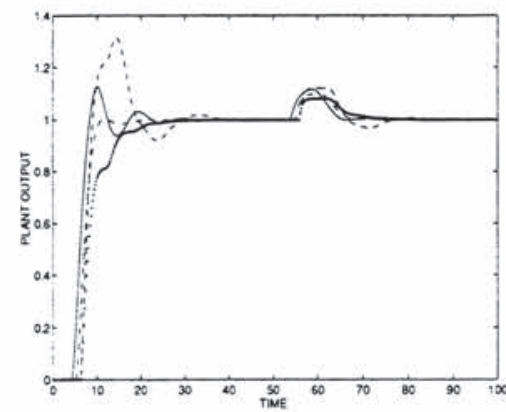

Fig. 5. Output for diferent plant uncertainties

Example 2: unstable plant In this example a plant with an integrator and long dead time is considered $P(s)=\frac{k}{s} e^{-t_{d} s}$. The model of the plant is the same as the one analysed in Matausek and 
Micic (1996) $\left(t_{d}=5\right)$ but with $k=2$ and using a sampling time $T=1$. It is desirable to have zero steady state for step references and an overshoot of less than $5 \%$. The obtained controller must guarantee robust stability for an error in the deadtime of two seconds.

Step 1: $R(z)$ is chosen as $R(z)=\frac{0.2 z}{z-0.8}$

Step 2: $D_{x}=1-z^{-1}$

Step 3: As $A_{s}=1$ and $l=1, D_{r} N_{x}-B N_{r} z^{-d}$ must have two roots in $z=1$. Then $N_{x}$ has two coefficients: $N_{x}=n_{1}+n_{2} z^{-1}$. In this case the obtained values are: $n_{1}=-16$ and $n_{2}=18$.

Step 4: Using the root locus $C(z)$ is computed in order to obtain two real poles for the closed loop, thus $C(z)=\frac{k_{c}\left(1-\alpha z^{-1}\right)}{1-z^{-1}}$ with $\alpha=0.95$ and $k_{c}=0.056$.

Step 5: To improve the obtained set point response $W$ is computed as $W=\frac{0.23\left(1-0.7 z^{-1}\right)}{1-0.93 z^{-1}}$.

The final control law is completed with:

$$
Q(z)=\frac{-16+14.8 z^{-1}+0.4 z^{-2}+0.4 z^{-3}+0.4 z^{-4}}{1-0.8 z^{-1}} .
$$

The closed loop performance is analysed in figure 6 for the nominal case (solid line). In order to make a comparative analysis a DTC is computed using the algorithm proposed in Matausek and Micic (1996) (with $K_{r}=0.17$ ) so as to obtain a similar nominal performance (see dashed lines in figure figure 6). At $t=0$ a step change in the reference is applied to the system and at $t=50 \mathrm{a}$ $5 \%$ step disturbance is introduced.

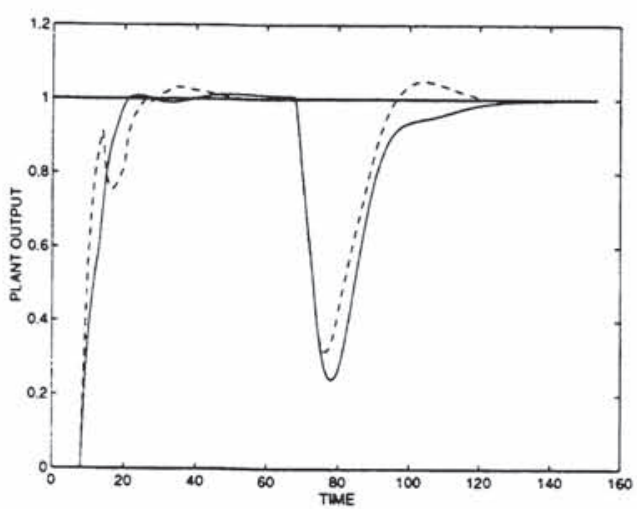

Fig. 6. Plant output for example 2

Step 6: Using $R, X$ and $C$ the norm-bound uncertainty (solid line) is compared to the unmodelled dynamics (delay error of $2 \mathrm{sec}$.) (dotted line) and to the norm-bound uncertainty of the controller proposed by Matausek and Micic (1996) (dashed line) in figure 7 . As can be seen, for a similar nominal performance, the proposed controller is more robust than the one proposed in Matausek and Micic (1996).

\section{CONCLUSIONS}

This paper presents a unified approach for designing a dead-time compensator that considers

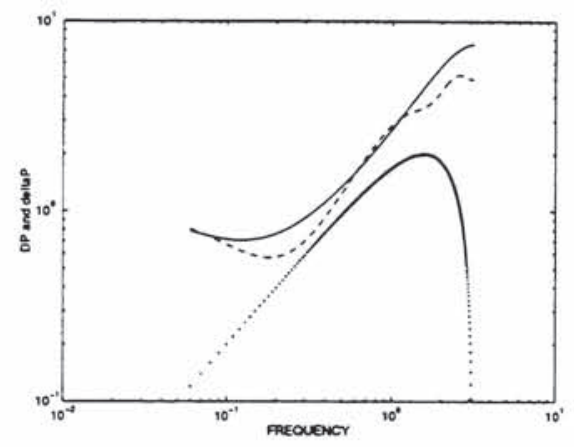

Fig. 7. Robustness analysis for example 2.

nominal performance and robustness of the resulting closed loop and can be used to control stable and unstable plants. The proposed structure uses the advantages of several dead-time compensators proposed in the literature and the obtained structure is simple. Some simulation results comparing the unified dead-time compensator with previous algorithms show the good qualities of the proposed controller.

\section{References}

K.J. Astrom, C.C. Hang, and B.C. Lim. A new smith predictor for controlling a process with a integrator and long dead-time. IEEE TAC, 39(2):343-345, 1994.

T. Furukawa and E. Shimemura. Predictive control for systems with time delay. Int. J. Control, 37:399-412, 1983.

T. Hagglung. An industrial dead-time compensating PI controller. Control Engineering Practice, 4(6):749-756, 1996.

H.P. Huang, C.L. Chen, Y.C. Chao, and P.L. Chen. A modified SP with an approximated inverse of dead time. AIChE journal, 36:1025-1031, 1990.

M.R. Matausek and A.D. Micic. A modified smith predictor for controlling a process with a integrator and long dead-time. IEEE TAC, 41(8):1199-1203, 1996.

M. Morari and E. Zafiriou. Robust Process Control. Prentice Hall, 1989.

J.E. Normey-Rico, C. Bordons, and E.F. Camacho. Improving the robustness of dead-time compensating P1 controllers. Control Engineering Practice, 5(6):801-810, 1997.

Z.J. Palmor. Robust digital dead time compensator controller for a class of stable systems. Automatica, 22: 587-591, 1986.

Z.J. Palmor. The Control Handbook: Chapters 10.8-10.10. CRC Press and IEEE Press, 1996.

Z.J. Palmor and Y. Halevi. On the design properties of multivariable dead time compensators. Automatica, 19: 255-264, 1983

Z.J. Palmor and D.V. Powers. Improved dead time compensator controllers. AIChE-Journal, 31:215-221, 1985.

C. Santacesaria and R. Scattolini. Easy tuning of smith predictor in presence of delay uncertainty. Automatica, 29:1595-1597, 1993.

M.J.O. Smith. Feedback Control Systems. Mc Graw Hill, 1958.

K. Watanabe and M. Ito. A process-model control for linear systems with delay. IEEE TAC, 26(6):1261-1269, 1981. 